\title{
Signal Processing System Mathematical Microscope
}

\section{Evgeni Nikolaevich Terentiev ${ }^{1}{ }^{*}$, Irina Igorevna Farshakova ${ }^{1}$, Irina Nikolaevna Prikhodko ${ }^{1}$, Nikolay Evgenievich Shilin-Terentyev ${ }^{2}$}

${ }^{1}$ M. V. Lomonosov Moscow State University, Faculty of Physics, Moscow, Russia

${ }^{2}$ EPAM Systems, Moscow, Russia

\section{Email address:}

en.teren@physics.msu.ru (E. N. Terentiev), irinafarshakova@gmail.com (I. I. Farshakova), prikhodko_in@mail.ru (I. N. Prikhodko), nikolay.terentyev@gmail.com (N. E. Shilin-Terentyev)

${ }^{*}$ Corresponding author

\section{To cite this article:}

Evgeni Nikolaevich Terentiev, Irina Igorevna Farshakova, Irina Nikolaevna Prikhodko, Nikolay Evgenievich Shilin-Terentyev. Signal Processing System Mathematical Microscope. Science Journal of Applied Mathematics and Statistics. Vol. 7, No. 5, 2019, pp. 71-78. doi: 10.11648/j.sjams.20190705.12

Received: June 26, 2019; Accepted: July 31, 2019; Published: October 15, 2019

\begin{abstract}
Conditionality is the main setting of Apparatus Function (AF) $O$ to increase resolution. The conditionality is numerically equal to the reciprocal of the minimum value of Modulation Transfer Function (MTF) $|\mathrm{M}(\mathrm{O})|$ or the magnitude of this gap. We introduce the magnitude SR of the estimating super-resolution. The concept of a Mathematical Microscope (MM) is formulated in this paper.
\end{abstract}

Keywords: Super-Resolution, Conditionality, Apodization in Inevertability, Modulation Transfer Function, Convolution, Fourier Transform

\section{Introduction}

If the MTF M (O) is limited: $0<\operatorname{diap} \leq|\mathrm{M}(\mathrm{O})| \leq 1$, then $1 \leq \mid \mathrm{M}$ $(\mathrm{R})|=1 /| \mathrm{M} \quad(\mathrm{O}) \mid \leq \mathrm{DIAP}$. The limitation in the frequency domain $\mathrm{DIAP}=1 /$ diap is the conditionality parameter of $\mathrm{AF}$ O.

If for even AF O DIAP we strive to unity, then we get AF DK - the delta Kronecker symbol - the most conditioned AF. It is clear that for in a good instrument the values of the MTF $\mathrm{M}(\mathrm{O})$ should be far away from zero.

Typically, the device is able to digitize images with a certain step, which is set by the developer of the device. We will consider this step as the unit $d x=1$. With step $d x=1$, the AF $\mathrm{O}$ is digitized too.

The main idea of the Super-resolution with the apodization reversing is that the sizes Loc and step digitation $d x$ of the $\mathrm{AF} O \mathrm{O}$ definition domain are chosen so that its inverse resolving function $\mathrm{R}=\mathrm{O}^{-1}$ on the selected discrete domain has the minimum norm.

The minimum of the norm $\mathrm{R}$ is realized by changing the digitization step $\mathrm{dx}$ and changing the length Loc of the domain of definition (or the size of Window function for) $\mathrm{AF}$ O.
In addition, we can coarsen the $\mathrm{AF} O$ to reversible $\mathrm{pO}=\mathrm{pR}^{-1}$ by decreasing its conditionality DIAP parameter [2-5].

The set of different lengths Loc of the domains of definition of the AF $\mathrm{O}$ with different steps of digitizing $\mathrm{dx}$ and different parameters DIAP will be denoted $\mathrm{LO}=\{\mathrm{pO} \mid[\mathrm{Loc}$, $\mathrm{dx}$, DIAP]\}.

From the set of LO, we take only those AF pO with min Nor (pR), i.e we choose operable devices with reversible AF $\mathrm{pO}=\mathrm{pR}^{-1}$.

Note that modern mathematical methods, when solving an inverse problem, essentially suggest using "a priori information about the smoothness" of signals (solutions of the inverse problem) on possibly no workable, defective devices.

The adequacy of the Discrete Measuring System (DMS) device is not checked. It is clear that "smooth solutions" are not quite adequate to associate with a defective device.

We can say that the concept of the Mathematical Microscope (MM) proposed in this paper is based on super-resolution with the apodization method.

A distinctive feature of using the apodization in invertability (reversing) technique is obtaining 
super-resolution of objects smaller than the pixel size of the original image.

\section{Problems of Setting of AF O}

The main task for choosing $\mathrm{AF} p \mathrm{pO}=\mathrm{pR}^{-1}$ is set as a minimum task [8-10]:

$$
\min _{\mathrm{LO}}\{\|\mathrm{pR}\| \mid \operatorname{Err}(\mathrm{pO}) \leq \mathrm{err}\}, \quad \mathrm{LO}=\{\mathrm{pO} \mid[\text { Loc }, \mathrm{dx}, 1 / \mathrm{mD}]\}
$$

with the Characteristics of the Adequacy of the Model (CAM) of AF O in SPS [8-10]:

$$
\{\mathrm{x}=\operatorname{Err}(\mathrm{pO}), \mathrm{y}=\operatorname{Nor}(\mathrm{pR}), \mathrm{z}=\mathrm{II}(\mathrm{pR} * \mathrm{O})\}
$$

Adjustable gap is bounded from above. Iz $<$ md $<\mathrm{mD}<1$, Iz is instrumental zero. In connection with this restriction, we consider the CAM AF $\mathrm{zO}$ on $\mathrm{LzO}=\{\mathrm{zO} \mid[$ Loc, $\mathrm{dx}, 1 / \mathrm{Iz}]\}$ variant taking into account the $\mathrm{Iz}$ inversion of the two-dimensional MTF M (O) (in the simplified notation for formulas without indices):

$$
\begin{gathered}
\mathrm{M}(\mathrm{zR})=\left\{\begin{array}{l}
1 / \mathrm{M}(\mathrm{O}), \text { for all }|\mathrm{M}(\mathrm{O})|>\mathrm{Iz} \\
\mathrm{M}(\mathrm{O})
\end{array}\right. \\
\{\mathrm{x}=\operatorname{Err}(\mathrm{zO}), \mathrm{y}=\operatorname{Nor}(\mathrm{zR}), \mathrm{z}=\mathrm{II}(\mathrm{zR} * \mathrm{O})\}
\end{gathered}
$$

In the CAM AF O $(2,4)$, the $\mathrm{z}$-axis values of the Indicators of Inversion II $\left(\mathrm{pR}^{*} \mathrm{O}\right)=(\mathrm{pR} * \mathrm{O})(0,0)$ and II $\left(\mathrm{zR}^{*} \mathrm{O}\right)=(\mathrm{zR} * \mathrm{O})$ $(0,0)$, i.e. convolution values of the adjustable $\mathrm{pR}$ and non-adjustable $\mathrm{zR}$ with the initial $\mathrm{AF} \mathrm{O}$ at the point $(0,0)$. In the reversible case II $\left(z R^{*} \mathrm{O}\right)=1, \mathrm{zR}=\mathrm{R}=\mathrm{O}^{-1}$. It may also happen that by changing the length of the domain of definition Loc, we get the reversible case II $\left(\mathrm{pR}^{*} \mathrm{O}\right)=1, \mathrm{pR}=$ $\mathrm{O}^{-1}$.

\subsection{Super-Resolution Function SR}

If the normalization of $\mathrm{AF} \mathrm{O}: \sum \mathrm{O}=1$ takes place, then at zero the $\operatorname{MTF} \operatorname{M}(O)(0,0)=1$, then in this case we will estimate the Super-Resolution by the expression:

$$
\mathrm{SR}=\frac{\sum \mathrm{M}(\mathrm{pR}) \mathrm{M}(\mathrm{O})}{\sum \mathrm{M}(\mathrm{pO})}
$$

Note that $\mathrm{SR} \geq 1$. If $\mathrm{AF} \mathrm{O}=\mathrm{DK}$, then $\mathrm{SR}=1$. The super-resolution expression $\mathrm{SR}$ is the function of the parameters [Loc, $\mathrm{dx}, 1 / \mathrm{mD}$ ] (1). It is desirable that the value of super-resolution does not depend on the size of images and large Loc. We will return to the SR function in section 4.1.

\section{2. $\mathrm{AF} \mathrm{pO}$ and zO Errors}

Error Err (pO) (and zO) we estimated [8-10] by the formula:

$$
\operatorname{Err}(\mathrm{pO})=\mathrm{SD}(\mathrm{O}-\mathrm{pO}) / \max (\mathrm{O}),
$$

where

$$
\mathrm{SD}(0-\mathrm{p} 0)=\operatorname{sqrt}\left(\sum(0-\mathrm{p} 0)^{2} /(\operatorname{Loc}+1)^{2}-1\right)
$$

$\operatorname{Err}(\mathrm{pO}) \operatorname{Err}(\mathrm{zO})$ are present in CAM AF pO, zO $(2,4)$.

\section{Original Images}

To illustrate the capabilities of the methods, three small fragments of the image of the surface of Mars with a stone will be taken.

Fragment of the image of the surface of Mars, presented on the grid with $d x=1$ and the interpolated image using FDST in a continuous case on the grid with $\mathrm{dx}=1 / 4$.

Note that the selected small fragments of images are low-contrast and all the brightness of fragment are represented by one byte. That means we have a low accuracy $2-4 \%$ image.

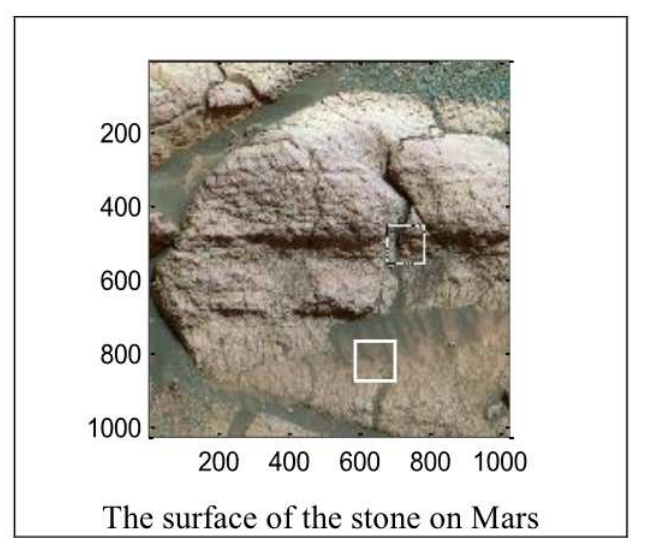

(a)

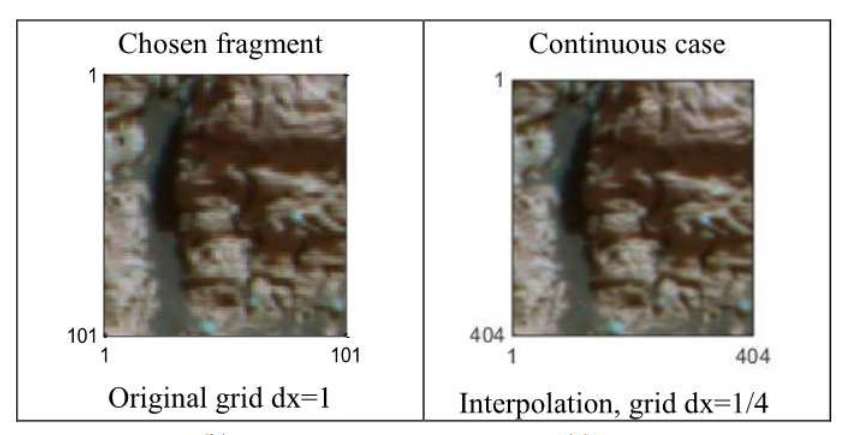

(b)

(c)

Figure 1. Initial Martian image (a), Chosen fragment (b) and the interpolated image (c).

\section{About Apodization Settings}

The word apodization in few dictionaries is defined as the method of extraction of hidden objects. In our case, this hidden object is discrete reversible AF $\mathrm{O}$ with a small value norm Nor $(\mathrm{R}), \mathrm{R}=\mathrm{O}^{-1}$.

Set the lengths of the domains of definitions of discrete $\mathrm{AF}$ $\mathrm{zO}$ set by array $\mathrm{Loc}=4: \mathrm{dx}: 12, \mathrm{dx}=1 / 4$.

On the set $\mathrm{AF} z \mathrm{O}: \mathrm{LzO}=\{\mathrm{zO} \mid[\mathrm{Loc}, \mathrm{dx}, 1 / \mathrm{Iz}]\}$, we build a CAM AF zO with Iz reversing $(3,4)$.

By the form of CAM AF $z O$, we see that all of them are reversible: $\mathrm{zO}=\mathrm{zR}^{-1}$, II $(\mathrm{zR} * \mathrm{O})=1$, $\operatorname{Err}(\mathrm{zO}) \sim 4.10^{-17}$.

The initial AF $\mathrm{zO}$ model with $\mathrm{dx}=1 / 4$ we cannot be considered as acceptable due to the very high response to noise, since $\lg ($ Nor $(z R))$ varies from 4.5 to $\sim 6$, see Figure 2.

We have a drawback, as a rule, one cannot use the $\mathrm{zR}$ 
functions because the response to noise turns out to be an unacceptably high $\lg$ (Nor (zR)).

However, we see that there are discrete options when the problem is solved by the ordinary reversion $\mathrm{pR}=\mathrm{pO}^{-1}$ for choosing options [Loc, $\mathrm{dx}, 1 / \mathrm{mD}$ ], where $1 / \mathrm{mD}$ is conditionality - main controlling parameter.

In essence, we propose an apodization (in invertability) technique for setting the $\mathrm{AF}$ according to the parameters of the set $\mathrm{LO}=\{\mathrm{pO} \mid[\mathrm{Loc}, \mathrm{dx}, 1 / \mathrm{mD}]\}$.

Mathematical focusing begins with a decrease in the DIAP conditionality (the DIAP value itself grows) or a decrease in the adjustable interval $\mathrm{mD}=1 / 5,1 / 10$, etc.

Further, we will analyze all this in a simplified version with a fixed length of the definition field Loc $=12$.

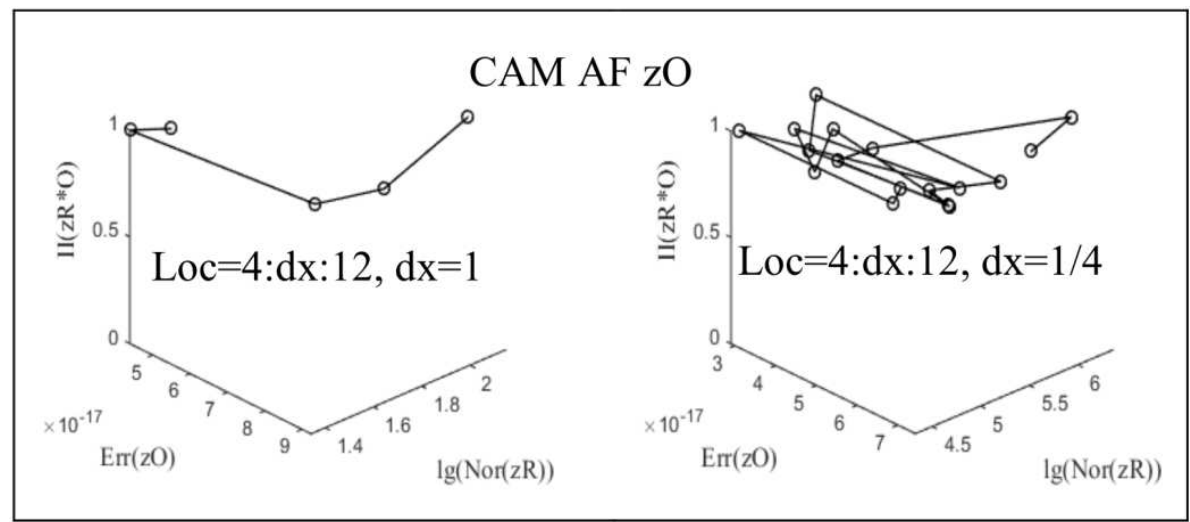

(a)

(b)

Figure 2. Noise reaction $\lg (\operatorname{Nor}(z R))$ in cases with $d x=1$ (a) and $d x=1 / 4$ (b).

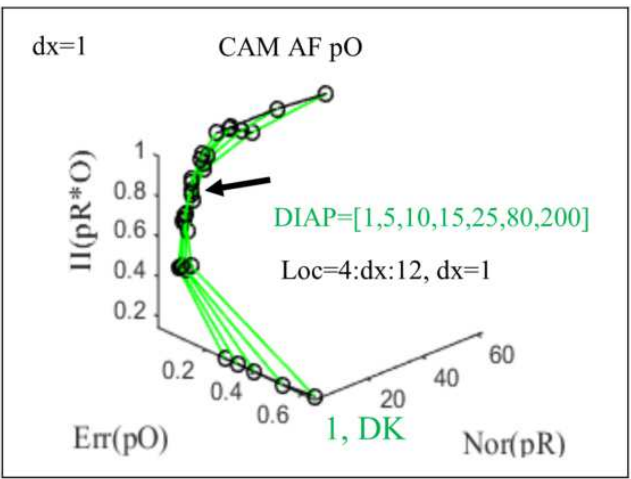

(a)

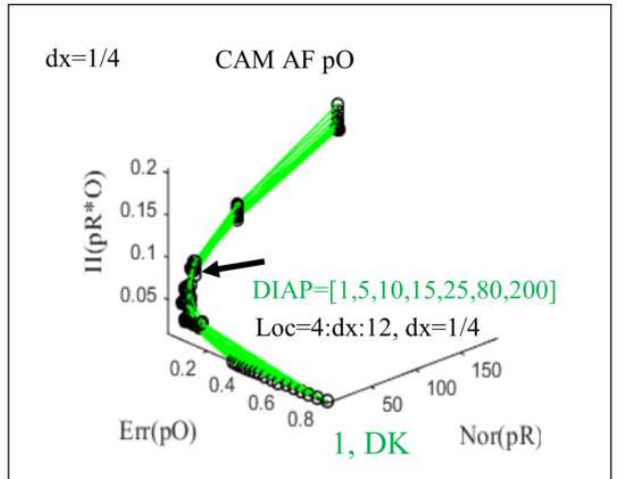

(b)

Figure 3. The apodization technique for conditionality setting the AF pO in cases with $d x=1$ (a) and $d x=1 / 4$ (b).

\section{CAM AF pO}

$\operatorname{Loc}=12, \mathrm{dx}=1$

Loc $=12, \mathrm{dx}=1 / 4$

Reversibility

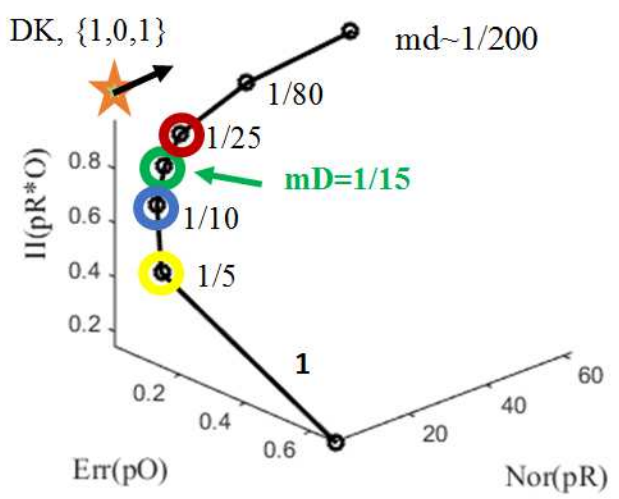

(a)

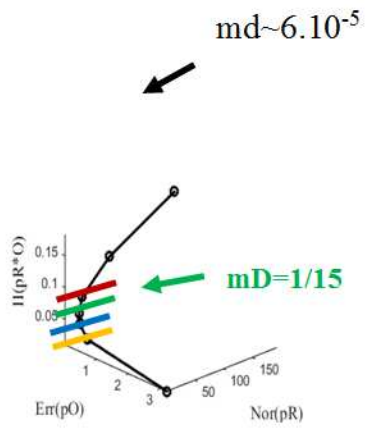

(b)

Figure 4. The gaps $m d=\min |M(O)|$ and $m D=\min |M(p O)|$ with $C A M A F p O$. 
With a decrease in $\mathrm{mD}$ (the conditionality of $\mathrm{AF} \mathrm{pO}$ decreases), the values of the super-resolution $\mathrm{SR}$ and the responses to the noise Nor (pR) increase, the errors Err (pO) decrease to zero, see Figures 4-6.

\subsection{About Values of SR Function}

In reversible cases, SR (5) as a function of three parameters [Loc, $\mathrm{dx}, 1 / \mathrm{mD}$ ] is a function with saturation by two parameters: the length of the domain of definition of Loc

$$
\operatorname{Loc}=4: \mathrm{dx}: 12 \text {, }
$$
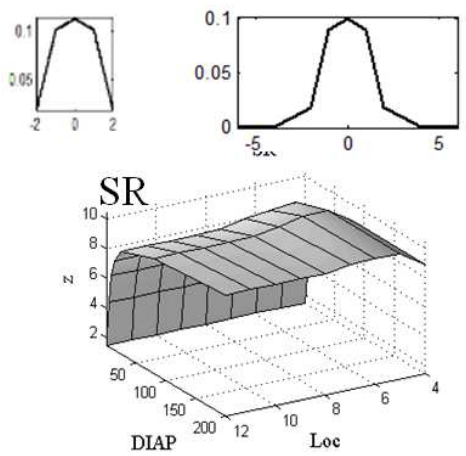

(a) and the conditionality DIAP. In the discrete case $d x=1$, this is seen in Figure 5(a) on the left. In the continuous case of $\mathrm{dx}=1 / 4$, this is evident in Figure 5(b) on the right for large values of DIAP in the case of Iz inversions. On the exact data we can get a high $\mathrm{SR} \sim 160$, see Figure $6(\mathrm{~b})$.

Moreover, since the image size, as a rule, is larger than the size of the Loc definition domain, the value (with saturation) of the conditioned super-resolution SR does not depend on the image size.

\section{$\operatorname{Loc}=4: \mathrm{dx}: 12$,}
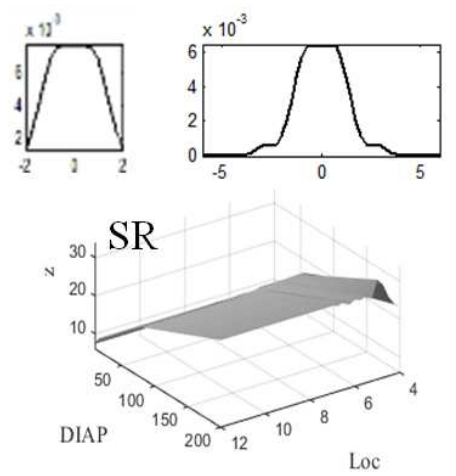

(b)

Figure 5. Examples of dependences of the SR super-resolution values on parameters [Loc, dx, $1 / \mathrm{mD}]$ in LO (4) that are tuned for local domains of definition of $A F \mathrm{P} O$ in discrete and continuous cases.

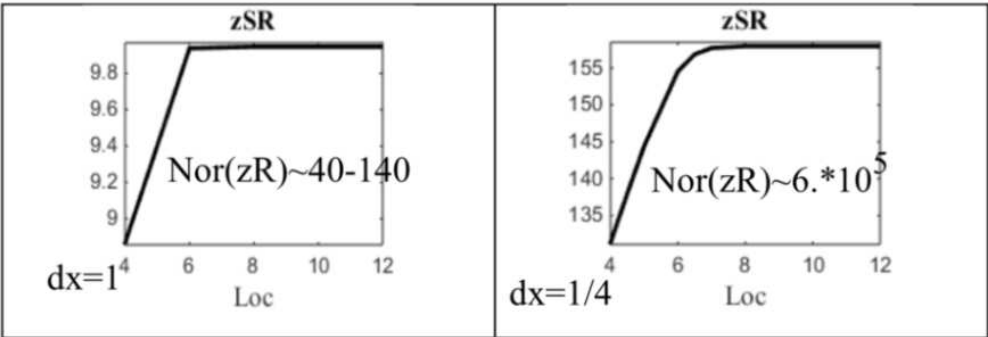

(a)

(b)

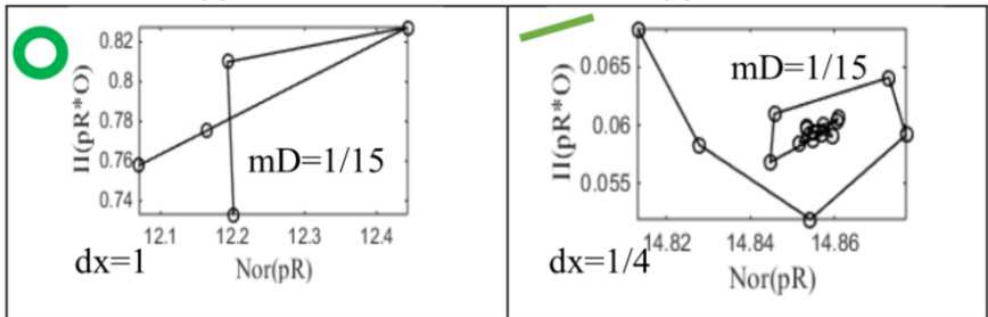

(c)

(d)

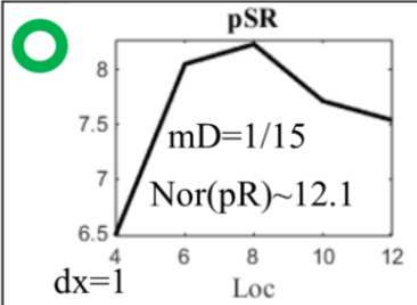

(e)

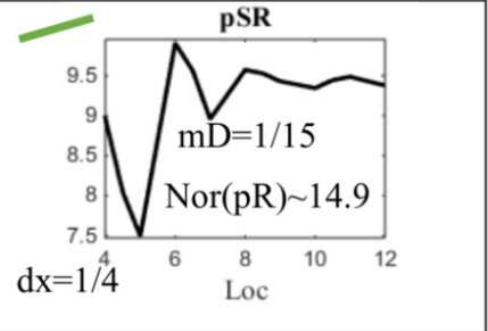

(f)

Figure 6. Dependences of the values of super-resolutions $p S R=S R$ and $z S R$ on $m D$, Loc in the discrete dx=1 and in the continuous $d x=1 / 4$ cases during Iz inversions. 


\subsection{Mathematical Focusing}

Mathematical focusing begins with a decrease in the:

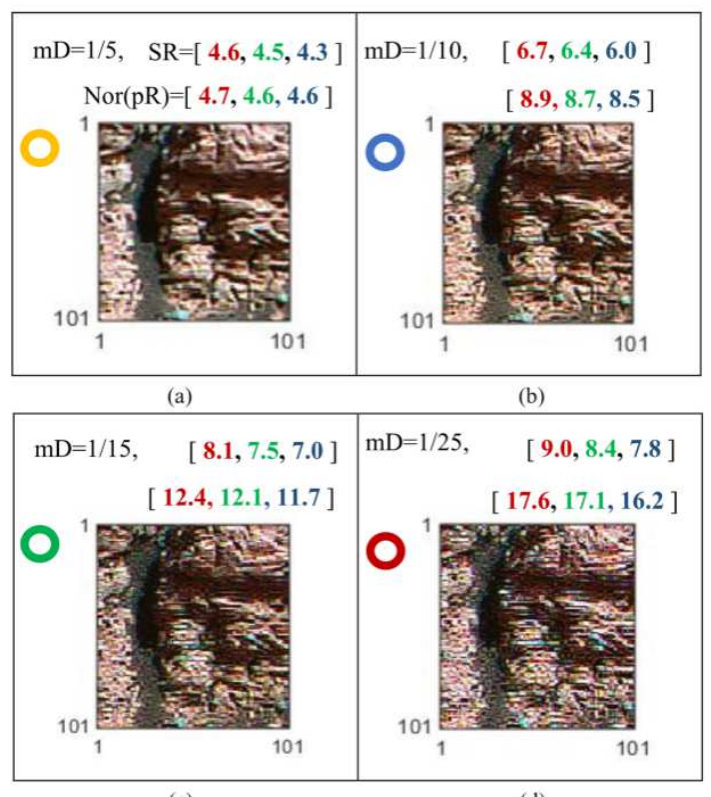

(c)

(d)

Figure 7. Conditioned super-resolutions SR and noise response Nor (pR) in the initial grid $d x=1$.

DIAP conditionality (the DIAP value itself grows) or a decrease in the adjustable interval $\mathrm{mD}=1 / 5,1 / 10$, etc.

Note that color images are usually of low accuracy, i.e. the brightness value is represented by one byte. For the first time, the super-resolution of color images is presented in the SPIE paper $[5,7]$. The CAM (2-4) first appeared in papers [8-10] when analyzing the operation of multibeams Antenna Patterns (a lot of beam comb).

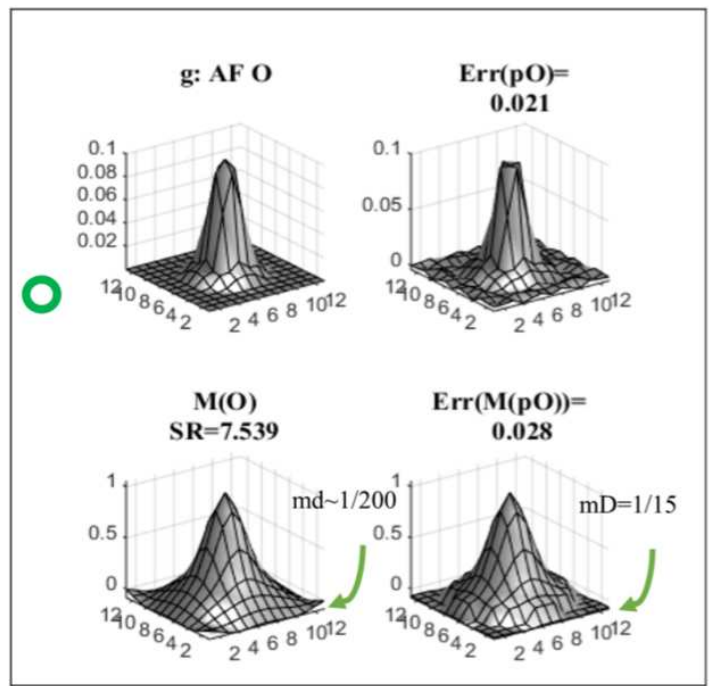

(a)

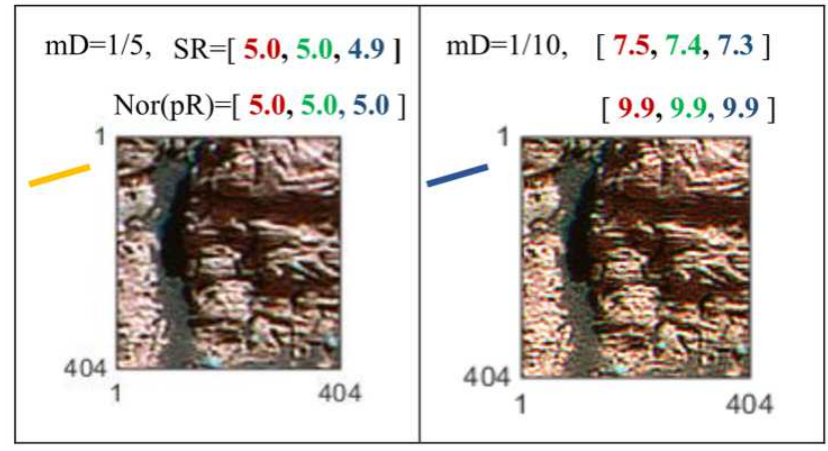

(a)

(b)

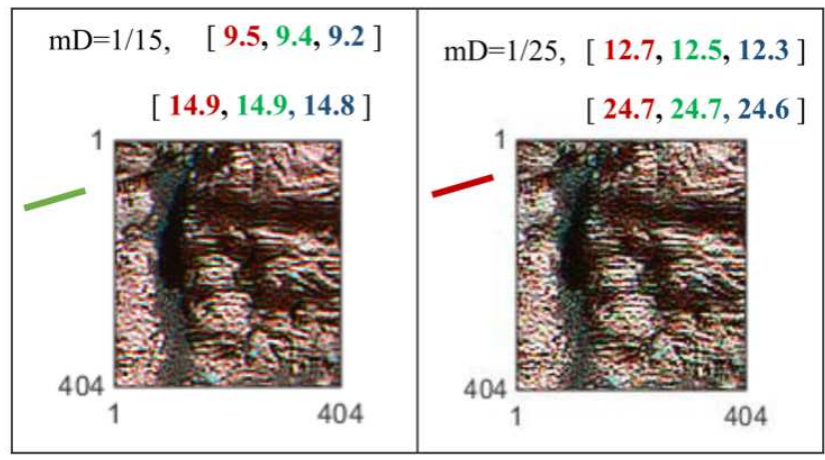

(c)

(d)

Figure 8. Conditioned SR super-resolutions and noise responses Nor $(p R)$ in case with interpolation $d x=1 / 4$.

\subsection{Gaps Md and Adjustable $\mathrm{mD}$}

Figure 4 shows the gaps $\mathrm{md}=\min |\mathrm{M}(\mathrm{O})|$ - not adjustable and $\mathrm{mD}=\min |\mathrm{M}(\mathrm{pO})|-$ adjustable gap between MTF $\mathrm{M}(\mathrm{O})$ and zero.

Figure 9. Discrete model of $A F p O$ with $d x=1$, gap $m d \sim 1 / 200$ and adjustable gap $m D=1 / 15$.

We believe that the controlling (md->mD) of AF O or the Antenna Pattern (AP) is very important for modern radar technologies. The goal of controlling $(\mathrm{md}->\mathrm{mD})$ is to improve the visual result or compensate for the effect of AP [14-17]. 


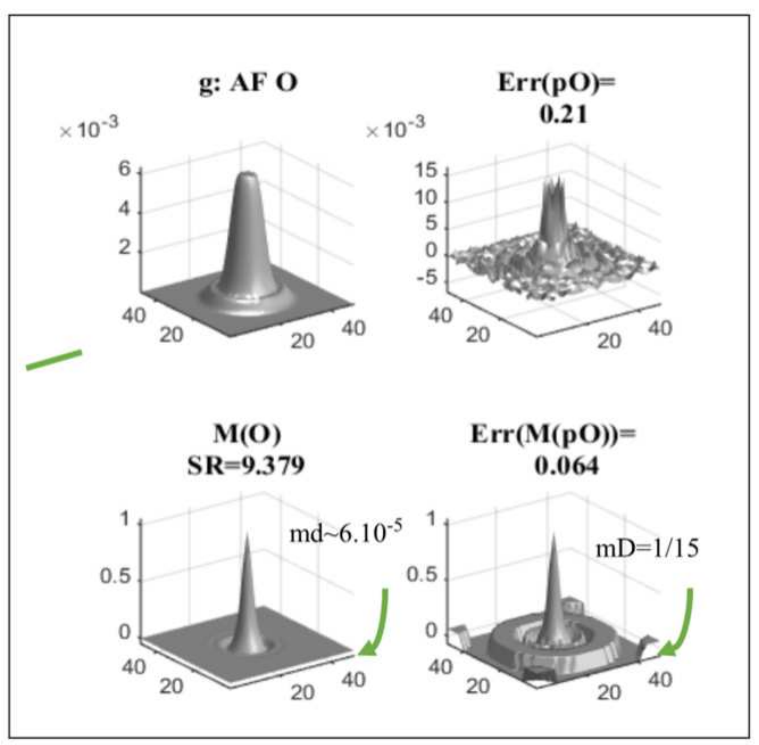

(a)

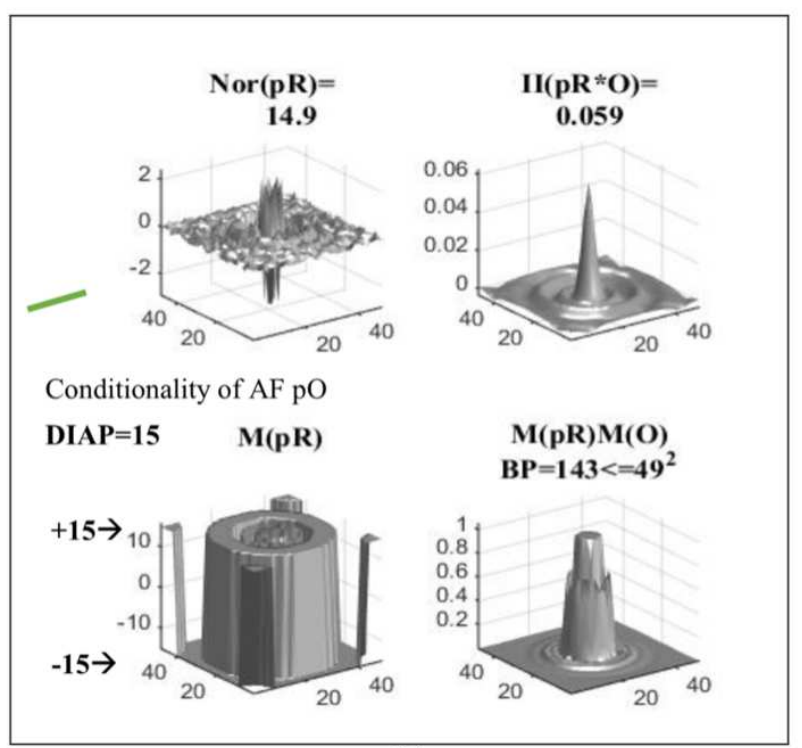

(b)

Figure 10. The continuous model of $A F$ pO with $d x=1 / 4$, gap $m d \sim 6.10^{-5}$ and adjustable gap $m D=1 / 15$.

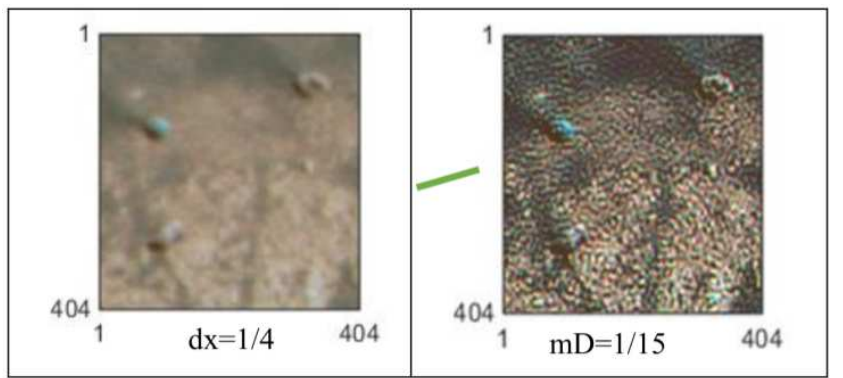

(a)

(b)

Figure 11. Super-resolved the sand grains.

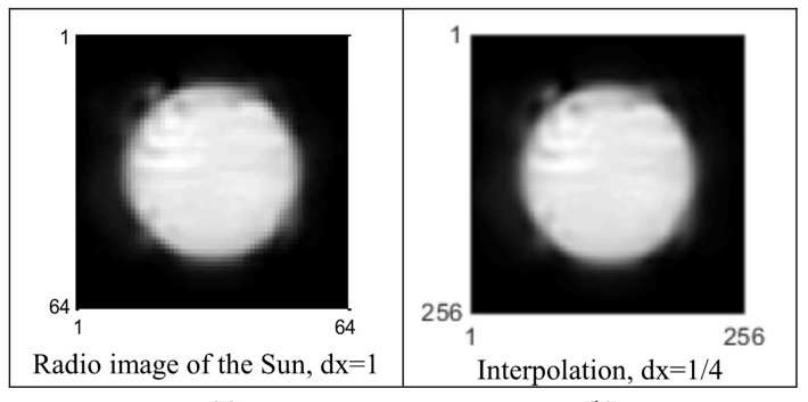

(a)

(b)

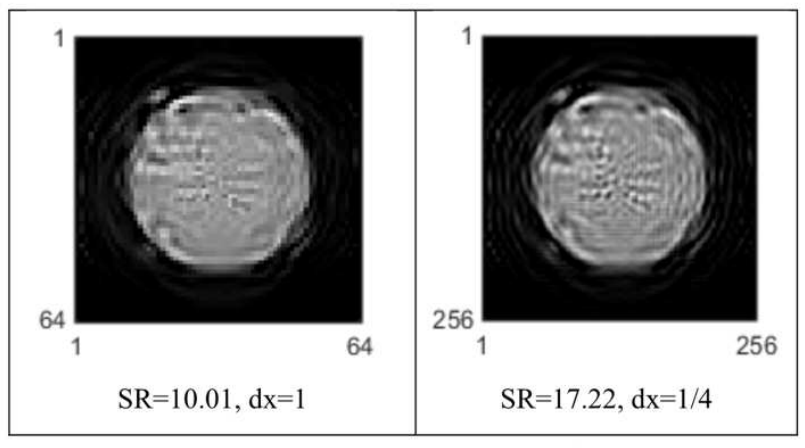

(c)

(d)

In this place of the article, we confess that we picked up $\mathrm{AF} \mathrm{O}=\{\mathrm{Or}, \mathrm{Og}, \mathrm{Ob}\}$ according to the resolution of the sand grains (in the gray dunes, see in Figure 1) in the lower area of the Martian stone. We believe that the result of super-resolution with conditionality $\mathrm{DIAP}=25$ is not acceptable, because the red color is knocked out. However, perhaps we are wrong and we should more carefully select the AF $\mathrm{O}$ in order to improve result of super-resolution.

\subsection{Conditional Super-Resolution in Radio-vision}

The antenna pattern of the radio-vision system is close to the AF of the Martian images, so we do not give it.

Below is the original radio image from a $3 \mathrm{~mm}$ wavelength telescope.

\subsection{CAM AF pO for SPS of Radio-vision}

The arrow denotes the places on CAM AF pO, which correspond to the images in Figure 1.

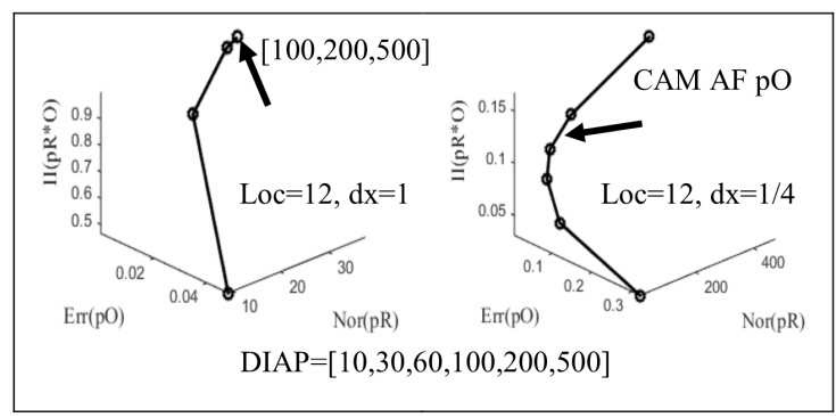

(a)

(b)

Figure 13. Examples of CAM AF pO in discrete and continuous cases only for $L o c=12$.

In Figure 14 below we study the behavior of the SR super-resolution values for $\mathrm{dx}=1 / 2$ and $1 / 8$.

Figure 12. Shows examples of super-resolution in the source grid $d x=1$ and with interpolation with $d x=1 / 4$. 


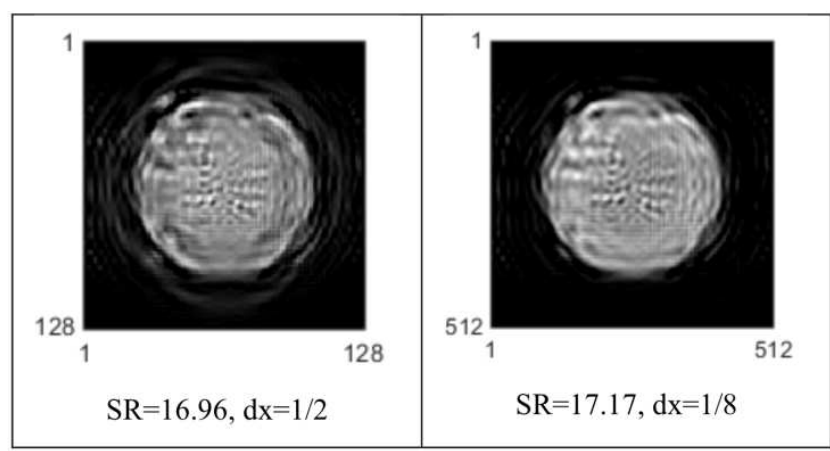

(a) (b)

navigators, etc. in future.

In our super-resolved images, jumps, differences in brightness, i.e. the solutions of the ill-posed inverse problem of compensating for distortion of $\mathrm{AF} \mathrm{O}$ are not smooth.

Interesting modifications of the methods [13-16] can be implemented in new radar technologies (with a controlled antenna pattern), in synthetic aperture radars, etc.

\section{Acknowledgements}

The work was done on its own initiative, without grant support.

\section{References}

MM in SPS is much more difficult to use than Tikhonov regularization [1]. In the regularization method, the residual error, which is obtained just due to a priori information about the smoothness of the solutions. In MM, in essence, a working AF is selected and nothing is assumed about the desired solution.

We can always return to the decision schemes using a priori information about their form. For example, we can smooth the solution with the assumption of a corresponding residual error. However, for most inverse problems this is not necessary, see comparison with regularization [11-12].

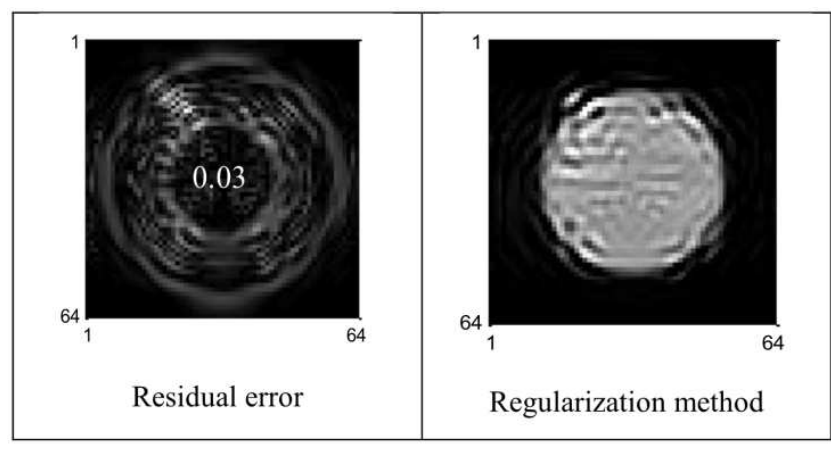

(a)

(b)

Figure 15. Residual error and the result obtained by the regularization method.

We are not sure that super-resolution methods are used in modern passive radio imaging systems. In passive radio vision, the super-resolution in Russia was first implemented in 1997-98; see the SPIE proceedings [1-4, 6].

\section{Conclusions}

For low-precision images (brightness are represented by one byte), achievable SR values are about 7-10, for two byte values - SR are about 17-20. We can use MM with any image-measuring devices: microscopes, telescopes, radars... We plan to use MM even in CT and MRT.

The regularization methods give smaller values of SR just because of the a priori smoothness of the solutions. Not smooth solutions allows us to widely use the MM SPS in high-precision control circuits of plasma, nuclear reactors,

[1] Tikhonov, A. N., Ufimtsev, M. V., Statistical processing of experimental results, Publishing house of Moscow University (1988) (in Russian).

[2] Yuri A. Pirogov, M. V. Lomonosov Moscow State Univ. (Russia); Magdy F. Attia, Johnson C. Smith Univ. (United States); Valeri V. Gladun, Andrey I. Dubina, Dmitri A. Tischenko, Evgeni N. Terentiev, M. V. Lomonosov Moscow State Univ. (Russia), Superresolution in millimeter-wave imaging technology, published in Proceedings Volume 3064: Passive Millimeter-Wave Imaging Technology, June 1997, Available on the SPIE Digital Library.

[3] Yuri A. Pirogov, M. V. Lomonosov Moscow State Univ. (Russia); Magdy F. Attia, Johnson C. Smith Univ. (United States); Isaiah M. Blankson, NASA Aerospace Research Div. (United States); Valeri V. Gladun, M. V. Lomonosov Moscow State Univ. (Russia); C. D. Papanicolopoulos, Georgia Tech Research Institute (United States); Dmitri A. Tishchenko, Evgeni N. Terentiev, Oksana A. Tarasova, M. V. Lomonosov Moscow State Univ. (Russia). Optimization of radiovision systems in millimeter-wave range, published in Proceedings Volume 3378: Passive Millimeter-Wave Imaging Technology II, August 1998, Available on the SPIE Digital Library.

[4] Yuri A. Pirogov, Valeri V. Gladun, Evgeni N. Terentiev, Dmitri A. Tischenko, Cheon W. Cho, Vladimir S. Ivanov, Moscow State Univ. (Russia), 3-mm wave range passive radio imaging system of high resolution, published in Proceedings Volume 4032: Passive Millimeter-Wave Imaging Technology IV, July 2000, Available on the SPIE Digital Library.

[5] Evgeni N. Terentiev, M. V. Lomonosov Moscow State Univ. (Russia); Nikolai E. Terentiev, XSIA (Russia); Fedor V. Shugaev, M. V. Lomonosov Moscow State Univ. (Russia), Ultraresolution of microwave, color, and synthetic color images, published in Proceedings Volume 5573: Image and Signal Processing for Remote Sensing X, November 2004, Available on the SPIE Digital Library.

[6] Evgeni N. Terentiev, Nikolay E. Terentiev, M. V. Lomonosov Moscow State Univ. (Russia), Applications of pointed ultra-resolution method in microwave imaging, published in Proceedings Volume 5789: Passive Millimeter-Wave Imaging Technology VIII, May 2005, Available on the SPIE Digital Library.

[7] Evgeni N. Terentiev, Nikolay E. Terentiev, M. V. Lomonosov Moscow State Univ. (Russia), Applications of pointed ultra-resolution method in colour imaging, published in Proceedings Volume 5817: Visual Information Processing XIV, May 2005, Available on the SPIE Digital Library. 
[8] Terentiev E. N., Terentyev N. E. CHARACTERISTICS OF ADEQUACY OF MODELS OF MEASURING COMPUTING SYSTEMS, Proceedings of the XIX International Forum on problems of science, technology and education, p. 95-97 (2015) (in Russian).

[9] Terentyev E. N., Terentyev N. E. PROBLEMS OF MULTI-BEAMS MEASURING - COMPUTING SYSTEMS, Proceedings of the XIX International Forum on problems of science, technology and education, p. 94-95 (2015) (in Russian).

[10] Terentiev E. N., Terentyev N. E. ADEQUATE SETTINGS OF A LOT OF BEAM COMB IN RADAR TECHNOLOGIES, Proceedings of the XIX International Forum on the problems of science, technology and education, p. 76-78, (2015) (in Russian).

[11] E. N. Terentiev, N. E. Terentyev MATHEMATICAL PRINCIPLES OF SETTING MEASURING-COMPUTING SYSTEMS AND REGULARIZATION, NOTES OF THE RAS, PHYSICAL SERIES, 2015, Volume 79, No. 12, p. 1633-1637 (in Russian).

[12] Terentiev, E. N., Terentiev, N. E.: Bulletin of the Russian Academy of Science. Physics, vol. 79, No 12, pp. 1427-1431 (2015) doi: 10.3103/S1062873815120229.
[13] E. N. Terentyev, N. E. Terentyev, Yu. A. Pirogov, I. I. Farshakova, Physical Principles for Setting Apparatus Functions of Measuring Instruments, SCIENTIFIC NOTES OF THE PHYSICAL FACULTY OF MOSCOW UNIVERSITY, 9 pp., No. 6, 1761005 (2017) (in Russian).

[14] E. N. Terentiev, N. E. Shilin-Terentiev, Physical and Mathematical Modeling of Earth and Environment Processes (2018), Classifiers in Super-Resolution Problems, pp. 441-455, Springer Proceedings in Earth and Environmental Sciences. Springer, Cham, doi.org/10.1007/978-3-030-11533-3_44.

[15] Terentiev E. N., Terentiev N. E., Farshakova I. I., PMMEEP 2017, Physical and Mathematical Modeling of Earth and Environment Processes, 19 Chapter, Principles of Controlling the Apparatus Function for Achieving Super-Resolution in images, pp. 171-182, Number of pages 382, Springer International Publishing, DOI: 10.1007/978-3-319-77788-7 19C.

[16] E. N. Terentyev, N. E. Shilin-Terentyev Conditional super-resolution with classifiers, SCIENTIFIC NOTES OF THE PHYSICAL FACULTY OF THE MOSCOW UNIVERSITY, No. 5 1850306, p. 1-9 (2018) (in Russian). 